\title{
FERM family proteins and their importance in cellular movements and wound healing (Review)
}

\author{
DAVID C. BOSANQUET, LIN YE, KEITH G. HARDING and WEN G. JIANG \\ Departments of Surgery and Wound Healing, Cardiff University School of Medicine, Heath Park, Cardiff CF14 4XW, UK
}

Received February 2, 2014; Accepted March 10, 2014

DOI: $10.3892 / \mathrm{ijmm} .2014 .1775$

\begin{abstract}
Motility is a requirement for a number of biological processes, including embryonic development, neuronal development, immune responses, cancer progression and wound healing. Specific to wound healing is the migration of endothelial cells, fibroblasts and other key cellular players into the wound space. Aberrations in wound healing can result in either chronic wounds or abnormally healed wounds. The protein 4.1R, ezrin, radixin, moesin (FERM) superfamily consists of over 40 proteins all containing a three lobed N-terminal FERM domain which binds a variety of cell-membrane associated proteins and lipids. The C-terminal ends of these proteins typically contain an actinbinding domain (ABD). These proteins therefore mediate the linkage between the cell membrane and the actin cytoskeleton, and are involved in cellular movements and migration. Certain FERM proteins have been shown to promote cancer metastasis via this very mechanism. Herein we review the effects of a number of FERM proteins on wound healing and cancer. We show how these proteins typically aid wound healing through their effects on increasing cellular migration and movements, but also typically promote metastasis in cancer. We conclude that FERM proteins play important roles in cellular migration, with markedly different outcomes in the context of cancer and wound healing.
\end{abstract}

\section{Contents \\ 1. Cancer and wound healing - similarities in cellular physiology and pathology \\ 2. Cellular migration and wound healing \\ 3. The FERM protein superfamily \\ 4. The FERM domain \\ 5. ERM proteins - background \\ 6. ERM proteins and cancer}

Correspondence to: Dr D.C. Bosanquet, Departments of Surgery and Wound Healing, Cardiff University School of Medicine, Heath Park, Cardiff CF14 4XW, UK

Email: davebosanquet@hotmail.com

Key words: cancer, cell migration, FERM domain, FERM proteins, wound healing
7. ERM proteins and wound healing

8. Protein 4.1R - background

9. Protein 4.1R in human disease and wound healing

10. Ehm 2 - background

11. Ehm 2 in human disease and wound healing

12. Conclusion

1. Cancer and wound healing - similarities in cellular physiology and pathology

Cellular migration is fundamental to both wound healing in health and cancer metastasis in disease. What separates these two diametrically opposed processes is the control and termination of this movement. When cellular migration is absent, wounded edges are stationary, resulting in chronic wounds; when migration is overactive, abnormal scarring results. In cancer, excessive cellular motility predisposes to metastasis, which is the cause of $90 \%$ of human cancer deaths $(1,2)$. Epithelial-mesenchymal transition (EMT) refers to the morphological changes observed during embryogenesis and foetal development, cutaneous wound repair and neoplastic invasion and metastases $(3,4)$. In response to wounding, keratinocytes at the wound edge are transformed from a sedentary to a migratory phenotype. They lose their cell-cell and cell-extracellular matrix (ECM) attachments, rearrange their actin cytoskeleton and express a variety of mesenchymal markers (such as vimentin) (5). However, in contrast to cancer progression, the changes observed in these wound edge keratinocytes are short lived and reversible (4). Maintaining this 'Goldilocks' type requirement of migration (not too much, not too little) is essential for health, and understanding which factors regulate such a process is of interest both to cancer biologists and wound healing scientists.

Historically, it was in the context of excessive inflammation, rather than excessive cellular migration, that the cross-over between cancer and wound healing was first recognised. As early as 1863, Virchow observed that excessive inflammation predisposed to cancer formation (6). Over 100 years later, Dolberg et al used an ingenious animal model to demonstrate this link (7). Chickens injected with the Rous sarcoma virus were observed to develop cancer not only at the site of injection, but also at distant body sites which were wounded. Furthermore, the application of an anti-inflammatory therapy inhibited cancer generation in these distant wounds (8). In his 
Table I. Inflammatory conditions known to predispose to malignant transformation.

Pre-malignant inflammatory condition

Cancer-associated outcome

(Refs.)

Barrett's oesophagus

Hepatic cirrhosis

Oesophageal cancer

(101)

Gastric ulceration

Hepatic cancer

Inflammatory bowel disease (ulcerative colitis)

Gastric cancer

Chronic pancreatitis

Colorectal cancer

Burn wound

Pancreatic cancer

Squamous cell carcinoma (SCC, Marjolin's ulcer)

landmark study, Dvorak succinctly suggested that tumours represent 'wounds that do not heal' (9).

Currently, the link between chronic inflammatory states predisposing to cancer formation is well established (Table I). Other links between wound healing and cancer include cell growth, angiogenesis and the formation of fibrous tissue/ ECM (10). These processes are appropriately regulated during physiological wound healing, whilst being uncontrolled in cancer. Of interest to our group is the regulation of cellular movements, in the context of both cancer metastasis and wound healing. During our search for key molecular markers involved in wound healing, we noted, as have other authors (11), marked similarities between molecular effectors of cancer and wound healing. Whilst this correlation is not absolute, there is a sufficient overlap to warrant further attention to this association (12). For example, Pedersen et al performed microarray analysis on keratinocytes during wound healing and compared this to keratinocytes from squamous cell carcinomas (SCCs) and normal tissue (13). They noted that keratinocytes from the wound edge transiently mimic their SCC transformed counterparts, with the most notable exception between the groups in gene expression profile relating to control of growth. Thus, keratinocytes captured whilst undergoing healing would share a number of similarities with their neoplastic counterparts. However, this overlap is not just confined to keratinocytes; fibroblasts also show remarkable parallels with their response to both wound healing and cancer progression. Chang et al cultured fibroblasts in serum, recreating a condition found only in the context of wound healing (14). They analysed the resultant gene expression profile, and showed a similar profile alteration in tumour-associated fibroblasts or tumour cells. Furthermore, the continued expression of this 'wound healing' genotype in breast, lung and gastric carcinomas predicts an increase in metastasis and death.

\section{Cellular migration and wound healing}

Common to all tissue repair processes is the migration of cells required for healing into the wound space, including endothelial cells (15), fibroblasts (16) and epithelial cells (17). Cells migrate through a process of 'crawling' motility, consisting of four well conserved steps: protrusion of the leading edge, adhesion to the substratum, de-adhesion and retraction of the rear (18). For directional movement, cells acquire a polarised morphology (19), responding to chemokines (chemotactic cytokines) or other extracellular signals. At the cell front (the 'leading edge'), actin assembles both lamellipodia and filopodia. It is these lamellipodia that adhere to the substratum and provide an anchor from which a force can be generated (20). Key to both lamellipodia and filopodia production is the assembly and protrusion of actin (20). Once a leading edge has been created, actin becomes polarised with a forward 'barbed' end and a backward 'pointed' end (both named due to myosin decoration of actin forming an arrow-head type pattern), and ATP fuels the disassembly of posterior actin filaments, and the assembly of anterior ones (21). Thus actin moves forward relative to the cell.

In order for these actin movements to be converted into cellular locomotion, there needs to exist a link between the cytoskeleton and the cellular membrane. The protein 4.1R, ezrin, radixin, moesin (FERM) family of proteins, so named as an acronym of its founding four members (protein 4.1R, ezrin, radixin and moesin), is a well described family involved in membrane-cytoskeletal interactions. We have already noted the important role FERM family proteins play in cancer progression (22). Herein we update and summarise these pertinent findings, and compare the effects of this protein family on wound healing and tissue repair.

\section{The FERM protein superfamily}

Members of the FERM superfamily are characterised by the presence of a conserved FERM domain at the N-terminus of the molecule, and often a spectrin/actin binding domain (SABD) at the C-terminus (23). Protein 4.1R, originally referred to as erythrocyte band protein 4.1 , was identified as an abundant protein in human erythrocyte ghosts, localising to the cytoskeleton and stabilising its shape, and the first of the FERM proteins to be identified (24-26). By 1991 three more FERM proteins were discovered; ezrin (27), radixin (28) and moesin (29) (also known as ERM proteins). Prior to the now commonly accepted title, the FERM domain has been known by a variety of names, including the $30 \mathrm{kDa}$ domain, $4.1^{\mathrm{N} 30}$, the membrane-cytoskeletal-linking domain, the ERM like domain, the ezrin like domain of the band 4.1 superfamily, the conserved $\mathrm{N}$-terminal region, the membrane attachment domain (23) and the talin, ezrin, radixin, moesin (TERM) domain (30).

The FERM family has grown considerably since this initial discovery of protein $4.1 \mathrm{R}$, with more than 40 members identified to date (31) (Fig. 1). On the basis of protein sequence similarity, this superfamily was divided in 1994 into five subgroups: band 4.1 proteins, ERM-related proteins, talinrelated molecules, protein tyrosine phosphatase (PTP) proteins and novel band 4.1-like 4 (NBL4) proteins (32). 


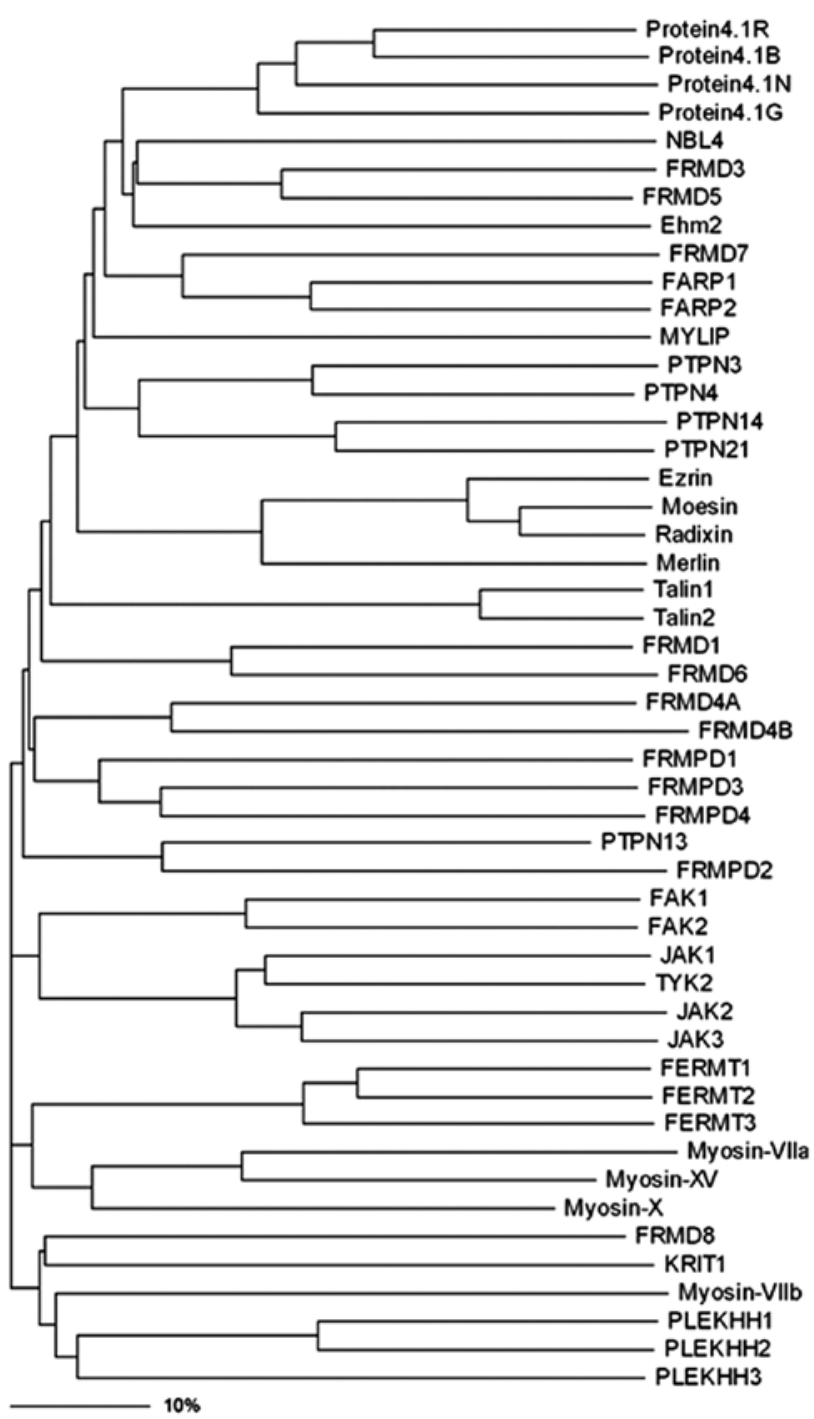

Figure 1. Phylogenic tree of the protein 4.1R, ezrin, radixin, moesin (FERM) family proteins. Phylogenetic analyses was performed using the ClustalW (http://www.ebi.ac.uk/clustalw/), and the dendrogram tree was drawn by using the Treeview (version 1.6.6, http://taxonomy.zoology.gla.ac.uk/rod/treeview. $\mathrm{html})$. The bar scale shows the identity shared by the proteins in their amino acid sequences. NBL4, novel band 4.1-like protein 4; FRMD, FERM domaincontaining protein; Ehm2, expressed in high metastatic cells 2; FARP, FERM RhoGEF and pleckstrin domain-containing protein; MYLIP, myosin regulatory light chain interacting protein; PTPN, protein-tyrosine phosphatase non-receptor-type; FRMPD, FERM and PDZ domain-containing protein; FAK, focal adhesion kinase; JAK, janus kinase protein; TYK, non-receptor tyrosine-protein kinase; FERMT, fermitin family homolog; KRIT, Krev interaction trapped protein; PLEKHH, pleckstrin homology domain-containing family $\mathrm{H}$ member.

\section{The FERM domain}

The FERM domain is a $30 \mathrm{kDa}, \mathrm{N}$-terminal, cysteine rich, basically charged, globular molecule, initially noted as it was resistant to mild chymotrypsin treatment (24). It is hydrophobic and contains numerous $\beta$-sheet structures, suggesting a tight, compact structure which may account for its resistance to proteolytic degradation (33). The FERM domain has been shown to consist of three lobes, the F1, F2 and F3 domains (also known as FERM-N, FERM-M and FERM-C subdomains), in a number of FERM proteins $(34,35)$. Pearson et al suggested that, although the FERM domain is composed of three smaller domains, these three function together as a single unit and are structurally rigid upon activation (36). The F1 and F2 domains appear to be the most highly conserved amongst the FERM domain proteins, whilst F3 encodes for more specific protein binding sites $(34,36)$. The N-terminal F1 lobe is structurally related to ubiquitin, the central F2 lobe is folded like an acyl-CoA binding protein and the C-terminal F3 subdomain is similar to a pleckstrin homology $(\mathrm{PH}) /$ phosphotyrosine-binding (PTB) domain (36). The FERM domain can bind a variety of proteins, lipids and molecules (Table II). Inactive FERM proteins are retained in the cytoplasm in a closed conformation, which masks transmembrane protein binding sites in both the FERM domain and the C-terminal region (36). Activation of the protein results in weakening of this interaction and subsequent exposure of the FERM domain and active $\mathrm{C}$-terminal region (Fig. 2). For example, when phosphatidylinositol-4,5-bisphosphate (PIP2) binds to the FERM domain of ezrin, its conserved C-terminal at $\mathrm{Thr}^{567}$ becomes phosphorylated, thus opening and activating the protein (37). Ezrin can then translocate to the membrane/ cytoskeleton interface, change its conformation, and undertake binding with various partners.

Certain FERM proteins have been well characterised with regards to human disease, cancer progression and wound healing. The following sections review the effects of certain key FERM proteins, ezrin, radixin, moesin, protein 4.1R and the novel protein expressed in highly metastatic cells 2 (Ehm2) in human disease and wound healing.

\section{ERM proteins - background}

Ezrin (cytovillin/p81/80k/villin-2), initially discovered in 1983 (38) as an $81 \mathrm{kDa}$ component of intestinal microvilli, is the most well characterised of these proteins and considered by some to be the prototype member of the ERM protein family due to its conserved wide spread distribution and highly homologous sequences of the FERM domain. Although ezrin (along with radixin and moesin) is expressed in most cultured cells, it has been shown to be highly concentrated in the gastrointestinal tract, lungs and kidneys, and is expressed in epithelial and mesothelial cells (39-41). Ezrin is localised to the juxta-membrane region and to soluble cytosolic pools. The dynamic localization between these two compartments is important in determining ezrin activity and is responsible for its cellular action $(42,43)$. Ezrin is involved in a myriad of cellular functions, such as cell adhesion, motility, apoptosis and phagocytosis.

Moesin (membrane-organising extension spike protein) was initially isolated from bovine uterus and characterised as a possible heparin receptor $(29,44)$. Moesin is concentrated in specialised microdomains, namely in the intracellular core of microextensions, such as filopodia, microvilli, microspikes and retraction fibers (45). Similar to ezrin, moesin is important in cellular motility and migration. Moesin is responsible for the formation of cortical actin complexes when activated (following phosphorylation of $\mathrm{Thr}^{558}$ ), and inducing actin depolymerisation and reassembly toward the cell membrane edge (46). In the context of cervical cancer, moesin-associated cellular motility has been shown to be upregulated by vascular 
Table II. The various binding partners of the N-terminal FERM domain.

\begin{tabular}{|c|c|c|}
\hline Class of FERM domain binding partner & Specific binding partner & (Refs.) \\
\hline \multirow[t]{2}{*}{ Membrane proteins } & Band 3 protein & $(107)$ \\
\hline & Glycophorin C and D & $(108,109)$ \\
\hline \multirow[t]{2}{*}{ Phosphoproteins } & p55 & $(107,109)$ \\
\hline & ERM-binding phosphoprotein 50 (EBP50) & $(110)$ \\
\hline \multirow[t]{4}{*}{ Adhesion molecules } & CD44 & $(111)$ \\
\hline & Inter-cellular adhesion molecule 1 (ICAM1) & $(112)$ \\
\hline & Inter-cellular adhesion molecule 2 (ICAM2) & $(112)$ \\
\hline & $\mathrm{Na}^{+} / \mathrm{H}^{+}$exchanger (NHE1) & $(113)$ \\
\hline Membrane lipids & PIP2 & $(114)$ \\
\hline Cytoplasmic proteins & Calmodulin & $(115)$ \\
\hline Other proteins & $\mathrm{Na}^{+} / \mathrm{H}^{+}$exchanger regulatory factor (NHERF) & $(116)$ \\
\hline
\end{tabular}

FERM, protein 4.1R, ezrin, radixin, moesin; PIP2, phosphatidyl inositol-4,5- bisphosphate.

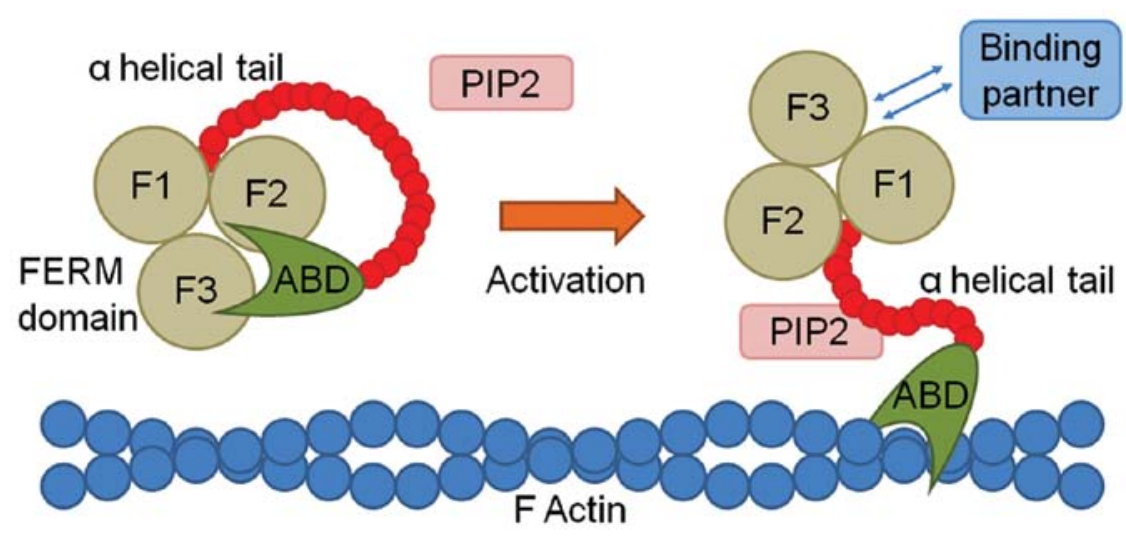

Figure 2. Schematic diagram demonstrating the activation and binding of protein 4.1R, ezrin, radixin, moesin (FERM) family proteins. The coiled FERM protein (left) is composed of three lobes (F1, F2 and F3), an $\alpha$ helical tail, and an actin binding domain (ABD), which is maintained in a closed conformation, and thus does not allow binding. On activation, in this instance by phosphatidyl inositol-4,5-bisphosphate (PIP2), the FERM protein opens, allowing the ABD to bind to $\mathrm{F}$ actin, and the FERM domain to bind to one of its various binding partners.

endothelial growth factor C (VEGF-C) and mediated by the RhoA/ROCK-2 signalling pathway (47).

In 1989 , radixin, an $82-\mathrm{kD}$ protein, was purified from a rat liver and shown to be a barbed end-capping protein associated with the undercoat of the cell-to-cell adherens junction (28). Subsequently, it was shown to be predominantly located to the hepatocyte microvilli (48). It shows high (>84\%) levels of sequence homology with ezrin and moesin (49). Radixin has been shown to interact with the $\mathrm{GABA}_{\mathrm{A}} \mathrm{R} \alpha 5$ subunit, a component of an inhibitory neurotransmitter receptor in neurons, at extra-synaptic sites (50). Thus, radixin may be indirectly important in modulating neuronal network activity.

\section{ERM proteins and cancer}

Over the years, more data has become available outlining the role ERM proteins play in cancer biology. Ezrin is the most studied protein in this context, whilst moderate work has been performed on moesin and very little on radixin. As FERM family members, and consistent with their role as membrane cytoskeletal linkers, ERM proteins typically promote cancer progression and metastasis (22). For example, the overexpression of ezrin results in the greater metastatic potential of osteosarcoma, hepatocellular carcinoma, breast cancer, lung cancer and pancreatic carcinoma (51-56). In the context of metastatic melanoma, ezrin deletion reduces important interactions with CD44, merlin and Lamp-1, and thus their recruitment (57). The resultant aberrant engagement with the surrounding microenvironment reduces cellular movements, thereby reducing the metastatic potential of these cells. When ezrin was knocked down in the CCR9-expressing acute T lymphocytic cell line, MOLT4, cell polarisation disappeared. Furthermore, E-cadherin translocation from the cytoplasm to the cell membrane was reduced, as was the invasive ability of the cells (58).

Ezrin also interacts with other proteins through which it has an effect on cancer. For example, the cytoskeletal-related protein, neurofibromatosis type 2 (NF2), acts as a cancer 
suppressor in the context of glioblastomas. However, ezrin has been shown to reduce NF2 expression via aberrant intracellular recruitment and thus intermolecular association. The resulting reduced levels of NF2 are observed in approximately one-third of glioblastoma cell lines and tumours (59).

Moesin has also been shown to promote metastasis in certain types of cancer. It has already been mentioned that moesin promotes cervical cancer metastasis through the RhoA/ROCK pathway (47). Greater levels of moesin (and also radixin) were found in pancreatic tumours which had metastasised to regional lymph nodes, compared to non-metastasising cancers (60). The knockdown of moesin using siRNA reduces the invasion of melanoma cells into 3D matrices, in part due to a loss of cellular polarisation (61).

\section{ERM proteins and wound healing}

Active ERM proteins have been examined using a phospho-ERM antibody, with affinity for ezrin, radixin and moesin, by a number of authors. For example, Jensen and Larsson observed that both CD44 and ERM proteins were co-localised to luminal F-actin domains in endothelial cells in vitro and in vivo (62). After wounding of the confluent monolayer of cells in vitro, focal $\mathrm{F}$-actin branching points appeared, with CD44 and ERM proteins again co-localised to this area, and associated with phosphorylated protein kinase (PK)C. The inhibition of PKC activity resulted in inhibited wound healing with reduced ERM-F-actin interaction and associated F-actin branching points. The authors concluded that ERM proteins, through the stimulation of PKC, play an important role in endothelial migration and thus, healing. These data compliment the work by $\mathrm{Ng}$ et al who showed that ERM protein phosphorylation by $\mathrm{PKC}$ improved in vitro wound healing in MCF7 breast cancer cells (63).

It is possible to study neuronal regeneration following injury in vitro using a neuron transection model. Haas et al initially showed that phosphorylated ERM proteins were localised to neuronal processes that formed several hours after neural transection, but were notably absent from relatively mature and stable cultured cells (64). They subsequently showed that the activation of ERM proteins was dependant on Rho kinase, with Rho kinase inhibition reducing phosphorylated ERM levels and subsequent response to wounding (65).

Tsuda et al showed that the signalling adaptor protein Crk associates with ERM proteins and enhances cell motility in 293T and 3Y1 (fibroblast) cells towards hyaluronic acid (66). Crk results in the translocation of ERM proteins to microvilli in the $3 \mathrm{Y} 1$ cell line, although this function was, as with the neuron transection model above, suppressed by Rho kinase. The authors concluded that Crk promotes cellular migration and motility in fibroblasts through its effects on ERM proteins.

Crepaldi et al utilised a kidney-derived epithelial cell line, LLC-PK1, to analyse the effect of ezrin overexpression on hepatocyte growth factor (HGF)-induced renal wound healing and epithelial migration (67). Cells overexpressing ezrin demonstrated enhanced migration and tubulogenesis in response to HGF compared to both a wild-type (WT) control, and a control overexpressing a truncated variant of ezrin, and thus concluded that ezrin promotes renal wound healing. The effects of moesin and lung injury were investigated using moesin knockout mice (68). These mice were compared to WT mice, and both had intra-tracheal bleomycin to simulate widespread lung injury. Moesin knockout mice developed more pronounced lung injury and fibrosis, following an abnormal cytokine and chemokine response, and had lower survival rates when compared to WT mice.

Moesin knockout mice were also used for investigating the role of moesin in hepatic injury (69). Hepatic stellate cells (HSCs) undergo activation in response to injury, and are important in hepatic wound healing and fibrosis. Response to hepatic wounding, caused by both common bile duct ligation and focal thermal denaturation, were compared in moesin knockout mice and WT controls. In moesin knockout mice inflammatory infiltrate, fibrosis and collagen deposition at the injury margin was markedly reduced compared to the WT mice. In vitro migration assay of moesin knockout HSCs demonstrated a significant reduction in migration compared to WT controls.

\section{Protein 4.1R - background}

Protein $4.1 \mathrm{R}$ is a $80 \mathrm{kDa}$ protein, initially found localised to the cytoskeleton on human erythrocytes, which binds to a variety of other proteins (adducin, tropomyosin, tropomodulin, dematin and protein p55 (70) and together stabilises the erythrocyte characteristic biconcave disc shape (71). Protein 4.1R consists of four structural domains (72); an N-terminal FERM domain, a $16 \mathrm{kDa}$ domain of unknown function, SABD and a $22 \mathrm{kDa}$ C-terminal domain which has been shown to interact with some proteins that form tight junctions, such as occludin, zonula occludens (ZO)-1 and ZO-2 (73). It is the most studied member of the protein 4.1 family, which consists of protein 4.1R (red blood cells), 4.1G (general), 4.1N (neuronal) and 4.1B (brain) (74).

A decrease in protein 4.1R expression due to chromosomal deletion results in hereditary elliptocytosis, which is characterised by abnormally shaped erythrocytes, haemolysis and splenomegaly (75). Abnormal erythrocyte architecture has been documented in protein 4.1R null mice (76). A similar model suggested that protein $4.1 \mathrm{R}$ deficiency results not only in the diminution of actin, but also in a reduction of various transmembrane proteins, including glycophorin C, XK, Duffy and $\mathrm{Rh}$, and results in the conformational change of band 3 and Kell epitopes (77). Protein 4.1R can also be deactivated by different methods. For example, in the presence of calcium, calmodulin decreases the affinity of protein 4.1R for the spectrin-actin complex, thus reducing the mechanical stability of the cellular membrane (78). Furthermore, protein 4.1R can be phosphorylated by a number of agents, including casein kinase, tyrosine kinase, PKA and PKC $(79,80)$. This phosphorylation reduces its ability to associate with spectrin approximately five-fold, with an increase in the relaxation of the cytoskeleton and associated cellular flexibility (81).

\section{Protein 4.1R in human disease and wound healing}

Although initially found to localise to human erythrocytes, it subsequently became clear that protein $4.1 \mathrm{R}$ is expressed in a variety of nucleated cells, is located in several different subcellular compartments, and is required for other diverse functions (82-85). Protein 4.1R has been shown to link with 
$\beta$-catenin in gastric epithelial cells, and such cells lacking protein 4.1R show impaired cell-cell contacts and disordered gastric glands (86).

The role of protein $4.1 \mathrm{R}$ in wound healing was recently investigated by Chen et al using protein $4.1 \mathrm{R}^{-/-}$mice (and their cultured keratinocytes) (87). Protein 4.1R deficient mice displayed delayed dermal wound healing. In vitro, protein $4.1 \mathrm{R}$ was shown to be present in the cytoplasm and the leading edge of moving cells. The absence of protein 4.1R resulted in reduced adhesion, spreading, migration and motility of keratinocytes. Focal adhesion complexes failed to localise in the absence of protein 4.1R. Furthermore, $\beta 1$ integrin levels were reduced, and shown to directly link to this protein. Of note, protein 4.1R deficient keratinocytes showed greater levels of $4.1 \mathrm{G}$ and $4.1 \mathrm{~N}$, without any change in $4.1 \mathrm{~B}$ levels, suggesting that these former two proteins compensate in part for the absence of protein 4.1R (87). Protein 4.1R has also shown to be important in the migration of endothelial cells. Ruiz-Sáenz et al showed that protein 4.1R interacts with Ras GTPase-activating-like protein 1 (IQGAP1), which acts as a scaffolding protein near the cell cortex at the leading edge of migrating cells, and both binds and cross-links actin filaments (88). The authors demonstrated that protein $4.1 \mathrm{R}$ was recruited to the leading edge of migrating cells, where it recruits and binds IQGAP1 through its FERM domain. The lack of protein 4.1R significantly slowed cellular migration.

\section{Ehm2 - background}

In 1996, Hashimoto et al identified four novel proteins in both high and low metastatic murine melanoma cells. Ehm2 was the second novel protein to be found in the highly metastatic (and notably absent in the low metastatic) K-1735 murine melanoma cell line, with an mRNA size of $4.0 \mathrm{~kb}(89)$. By the year 2000, the same team had cloned Ehm2 (90). The authors demonstrated that the Ehm 2 gene encodes a protein with 527 amino acids, with a $41 \%$ amino acid identity with the FERM domain. Further analysis revealed that Ehm 2 showed the greatest homology with NBL4, and was thus classified in the NBL4 subfamily. A human homologue, with significant homology (83\% identity, mapped to chromosome 9) was identified, along with seven rat clones and one pig clone, again with significant homology (83-92\%), suggesting that Ehm2 is a highly conserved gene (90).

In 2003, Chauhan et al characterised Ehm2 in a human fibrosarcoma cell line model (HT-AR1 cells) of steroid-induced cytoskeletal reorganisation, and demonstrated that Ehm 2 was androgen (dihydrotestosterone)-, but not dexamethasone-regulated (91). They analysed Ehm 2 mRNA levels in a variety of human tissues, and found the expression of a $3.8 \mathrm{~kb}$ transcript (isoform 1), which was expressed in greater levels in the testis, with a lower expression in prostate and breast tissue. An additional $2.3 \mathrm{~kb}$ Ehm 2 transcript was also found in these three tissues, with their transcripts only differing in the length of the 3' untranslated region (3'UTR). In addition, the human brain contained high levels of a $5.6 \mathrm{~kb}$ Ehm 2 transcript, encoding a protein of 913 amino acids (isoform 2), with an additional 409 amino acids of unknown function. Further analysis of the Ehm 2 protein revealed that it appeared to encode only an extended FERM domain located 70 amino acids from the N-terminus. Unlike other FERM domain proteins, the $\mathrm{C}$ terminus did not appear to encode any additional protein function. The authors subsequently hypothesised that Ehm2 either encodes a novel activity, or possibly, functions as a dominant negative modulator of other FERM proteins (92). Phylogenetic tree analysis revealed that Ehm2 belongs to a subfamily consisting of at least nine members from seven different species, including the Drosophilia Yurt gene (93).

\section{Ehm 2 in human disease and wound healing}

Wang et al investigated the role of Ehm 2 in prostate cancer. Ehm2 mRNA and protein levels were higher in immortalised prostate cancer cell lines (LNCaP, PC-3 and LAPC4) compared with the immortalised prostate epithelial cell line (PNT1a) (94). Similarly, Ehm 2 mRNA levels, as analysed by qRT-PCR, were significantly greater in prostate cancer tissues compared to benign controls. Immunohistochemical (IHC) analysis confirmed a significantly greater expression of Ehm2 protein in prostate cancer tissues compared with benign controls, with cancers associated with PSA recurrence following radical prostatectomy having a significantly greater staining compared to those without recurrence. Using transient transfection and siRNA knockdown in PNT1 and LAPC4 cells respectively, the authors demonstrated that increased Ehm 2 levels were associated with decreased adhesion to collagen IV. Schulz et al subsequently confirmed that Ehm2 mRNA levels were significantly increased in cancerous compared to non-cancerous prostate samples, and also showed that higher levels of Ehm2 expression (mRNA) significantly increased the likelihood of biochemical recurrence (95). Other authors have used microarray technology to show that Ehm 2 transcript levels are greater in neoplastic prostate samples (96-98).

Yu et al recently demonstrated that elevated levels of Ehm2 mRNA and protein were found in breast cancer tissues compared to controls, analysed with qRT-PCR and IHC (99). Higher levels were observed in node-positive disease (vs. node-negative), more advanced disease [TNM (tumour, node, metastasis)-4 disease vs. TNM1-3], higher levels of the Nottingham prognostic index (a marker of prognosis) and evidence of metastasis. There was a significant reduction in disease-free survival in patients with high levels of Ehm2. In vivo data using MCF7 Ehm 2 knockdown cells showed Ehm 2 enhanced growth and invasion; however, there was no effect on motility or adhesion. Ehm 2 knockdown was associated with the reduced mRNA and protein expression of MMP9.

We recently demonstrated that Ehm2 is upregulated in samples obtained from the periphery of acute healing wounds compared to chronic non-healing wounds (100). Furthermore, chronic wounds which subsequently healed displayed a greater cellular staining for Ehm2 than those which failed to heal, which was noted particularly at the leading keratinocyte edge of the healing wounds (Fig. 3A). In order to investigate the mechanisms behind this observation of improved wound healing, we created a stable Ehm 2 knockdown in the HaCaT keratinocyte cell line. In vitro experiments revealed that Ehm 2 knockdown slowed cellular adhesion, migration and motility (Fig. 3B-D), without affecting cellular growth, apoptosis or cell cycle rates. These data suggest that Ehm2 acts as a pro-migratory protein, in common with other FERM family proteins, and promotes wound healing through the process of re-epithelialisation. 
A

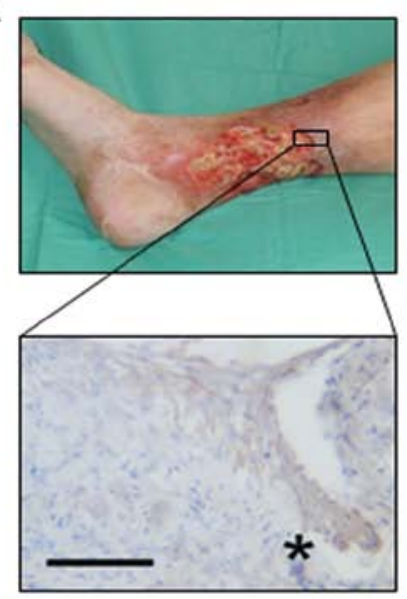

Ehm2 in keratinocytes

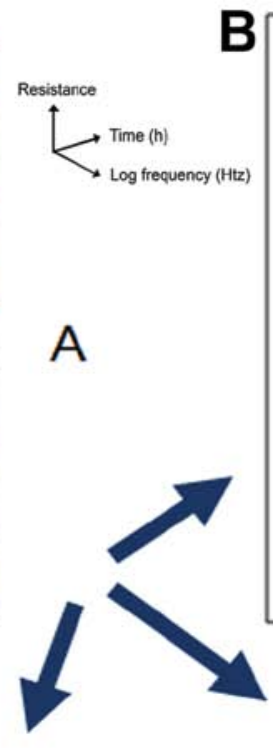

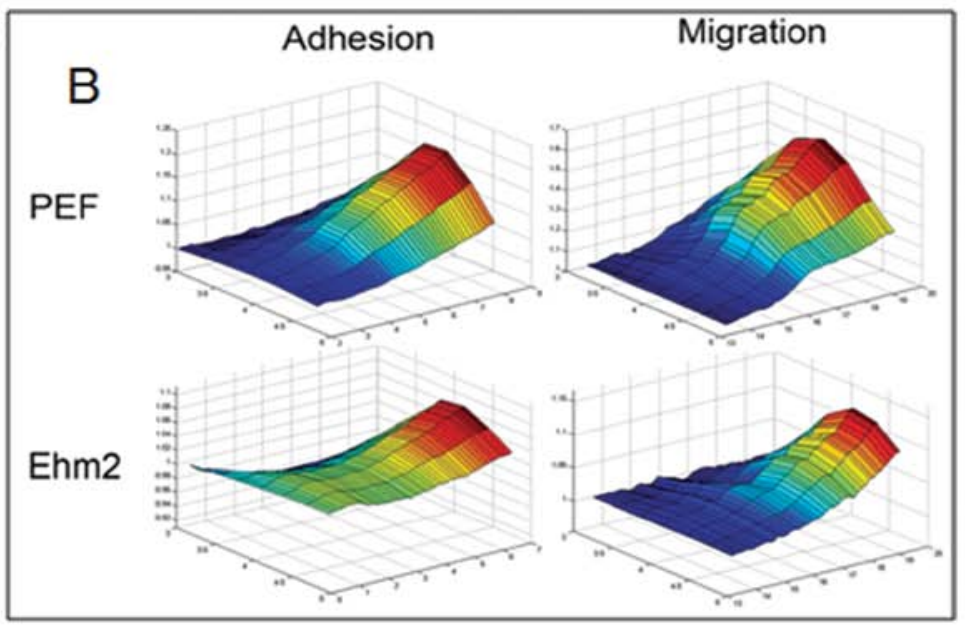

Electrical cell substrate impedance (ECIS) testing

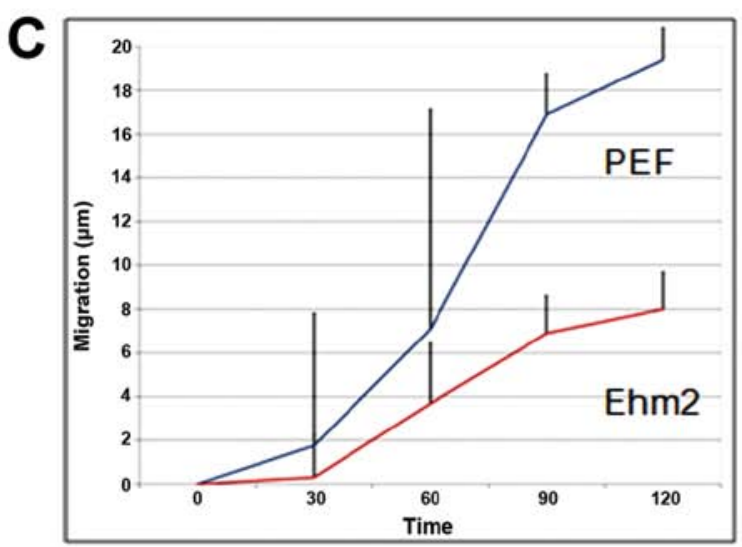

Scratch assay

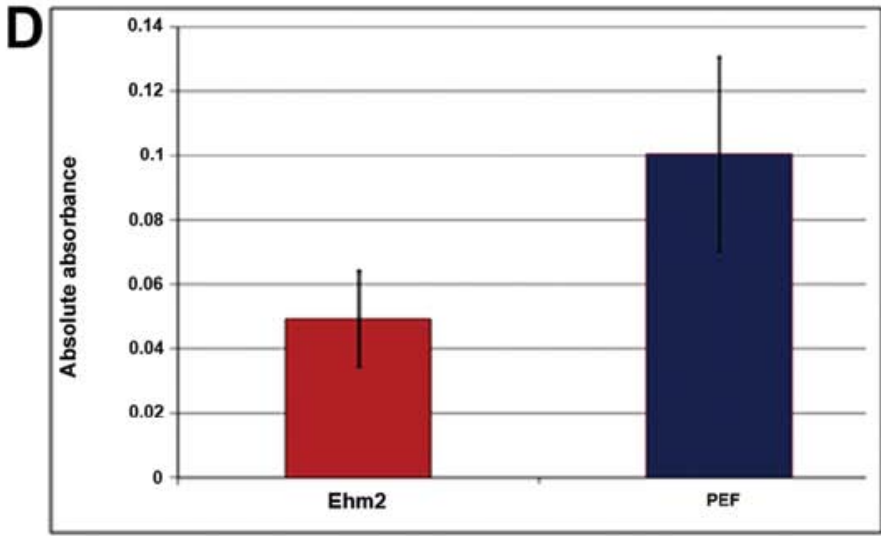

Motility assay

Figure 3. Example of data relating to the protein 4.1R, ezrin, radixin, moesin (FERM) protein expressed in high metastatic cells 2 (Ehm2) in wound healing. (A) Biopsies [immunohistochemical (IHC) staining, x100 magnification; line represents $0.1 \mu \mathrm{m}$ ] taken from the periphery of healing chronic wounds showed greater staining of Ehm2 in keratinocytes than those that were non-healing, particularly noted in the leading edge of keratinocytes as they migrated over the wound bed (*). (B-D) Subsequent in vitro experiments revealed that Ehm2 knockdown in a HaCaT keratinocyte model significantly slowed cellular motility in a variety of models compared to plasmid controls. (B) 3D data created by the electric cell-substrate impedance sensing (ECIS ${ }^{\mathrm{TM}}$ ) software used to analyse cellular adhesion and migration. Shown is a typical individual 'run' in both the Ehm2 knockdown (labelled 'Ehm2') and plasmid cell line showing that Ehm2 knockdown slows both adhesion and migration compared to plasmid control. (C) Results from 3 repeats (means \pm SEM) of a standard scratch/wounding assay comparing the wound healing rates of Ehm2 knockdown (red) and plasmid control (blue) cell lines at 30 min intervals. Migrated distances were significantly lower as measured at 90 and $120 \mathrm{~min}$ (p<0.01, Student's t-test). (D) Analysis of beads motility assay of Ehm2 knockdown (labelled Ehm2) and plasmid control cell lines. Results shown are the means $( \pm$ SEM) of 3 repeats. Ehm2 knockdown results in a marked reduction in motility (with absorbance used as a proxy measurement of cellular number, measured using a spectrophotometer) when compared to the plasmid control ( $\mathrm{p}=0.15$, 2-tailed Student's $\mathrm{t}$-test). Images derived from Bosanquet et al (100), used with permission from the Journal of Dermatological Science.

\section{Conclusion}

The migration of cells is essential not only for wound healing, but also for embryonic development, neuronal development and immune responses, and is also crucial for cancer metastasis. This brief review highlights the role that certain FERM proteins, the ERM proteins, protein $4.1 \mathrm{R}$ and Ehm2, play in wound healing and other diseases. Although there is some heterogeneity amongst this family, the FERM proteins generally promote the migration of cells, such as keratinocytes and endothelial cells, through their role as mediators between the cytoskeleton and the cell membrane, linking to a large number of different receptors. It is suggested that it is through these various mechanisms (i.e., re-epithelialisation and angiogenesis) that wound healing occurs.

This positive wound healing effect has been noted across a variety of models and in differing cell types. However, whilst in vivo results can be suggestive of an important role within wound healing, evidence corroborated with in vivo tissue data, or animal models, are often lacking. Whilst this does not necessarily detract from the conclusions, it highlights the need for further studies to fully assess the significance of FERM protein alterations in human tissue repair and regeneration. 


\section{Acknowledgements}

The authors wish to thank the Welsh Government (A4B scheme) for supporting this study.

\section{References}

1. Weigelt B, Peterse JL and van 't Veer LJ: Breast cancer metastasis: markers and models. Nat Rev Cancer 5: 591-602, 2005.

2. Mehlen P and Puisieux A: Metastasis: a question of life or death. Nat Rev Cancer 6: 449-458, 2006.

3. Yang J and Weinberg RA: Epithelial-mesenchymal transition: At the crossroads of development and tumor metastasis. Dev Cell 14: 818-829, 2008.

4. Arnoux V, Come C, Kusewitt D, Hudson L and Savagner P: Cutaneous Wound Reepithelializaton: A partial and reversible EMT. In: Rise and Fall of Epithelial Phenotype: Concepts of Epithelial-Mesenchymal Transition. Savagner P (ed). Springer, Berlin, pp111-134, 2005.

5. Yan CL, Grimm WA, Garner WL, et al: Epithelial to mesenchymal transition in human skin wound healing is induced by tumor necrosis factor-alpha through bone morphogenic protein-2. Am J Pathol 176: 2247-2258, 2010.

6. Virchow R (ed): Aetiologie der neoplastischen Geschwulste/ Pathogenie der neoplastischen Geschwulste. In: Die Krankhaften Geschwülste. Verlag von August Hirschwald, Berlin, pp57-101, 1863.

7. Dolberg DS, Hollingsworth R, Hertle $\mathrm{M}$ and Bissell $\mathrm{MJ}$ : Wounding and its role in RSV-mediated tumor formation. Science 230: 676-678, 1985.

8. Martinsgreen M, Boudreau $\mathrm{N}$ and Bissell MJ: Inflammation is responsible for the development of wound-induced tumors in chickens infected with Rous sarcoma virus. Cancer Res 54 4334-4341, 1994.

9. Dvorak HF: Tumors: wounds that do not heal. Similarities between tumor stroma generation and wound healing. N Engl J Med 315: 1650-1659, 1986.

10. Schafer M and Werner S: Cancer as an overhealing wound: an old hypothesis revisited. Nat Rev Mol Cell Bio 9: 628-638, 2008.

11. Antsiferova $\mathrm{M}$ and Werner S: The bright and the dark sides of activin in wound healing and cancer. J Cell Sci 125: 3929-3937, 2012

12. Grose R: Common ground in the transcriptional profiles of wounds and tumors. Genome Biol 5: 228, 2004.

13. Pedersen TX, Leethanakul C, Patel V, et al: Laser capture microdissection-based in vivo genomic profiling of wound keratinocytes identifies similarities and differences to squamous cell carcinoma. Oncogene 22: 3964-3976, 2003.

14. Chang HY, Sneddon JB, Alizadeh AA, et al: Gene expression signature of fibroblast serum response predicts human cancer progression: similarities between tumors and wounds. PLoS Biol 2: 7, 2004

15. Eming SA, Brachvogel B, Odorisio T and Koch M: Regulation of angiogenesis: wound healing as a model. Prog Histochem Cytochem 42: 115-170, 2007.

16. Midwood KS, Williams LV and Schwarzbauer JE: Tissue repair and the dynamics of the extracellular matrix. Int J Biochem Cell Biol 36: 1031-1037, 2004.

17. Martin P: Wound healing--aiming for perfect skin regeneration Science 276: 75-81, 1997

18. Pollard TD and Borisy GG: Cellular motility driven by assembly and disassembly of actin filaments. Cell 112: 453-465, 2003.

19. Mellman I and Nelson WJ: Coordinated protein sorting, targeting and distribution in polarized cells. Nat Rev Mol Cell Biol 9: 833-845, 2008

20. Small JV, Stradal T, Vignal E and Rottner K: The lamellipodium: where motility begins. Trends Cell Biol 12: 112-120, 2002.

21. Bugyi B and Carlier MF: Control of actin filament treadmilling in cell motility. Annu Rev Biophys 39: 449-470, 2010.

22. Yu H, Zhang Y, Ye L and Jiang WG: The FERM family proteins in cancer invasion and metastasis. Frontiers in bioscience: a journal and virtual library 16: 1536-1550, 2011.

23. Chishti AH, Kim AC, Marfatia SM, et al: The FERM domain: a unique module involved in the linkage of cytoplasmic proteins to the membrane. Trends Biochem Sci 23: 281-282, 1998.

24. Leto TL and Marchesi VT: A structural model of human erythrocyte protein 4.1. J Biol Chem 259: 4603-4608, 1984.
25. Tyler JM, Hargreaves WR and Branton D: Purification of two spectrin-binding proteins: biochemical and electron microscopic evidence for site-specific reassociation between spectrin and bands 2.1 and 4.1. Proc Natl Acad Sci USA 76: 5192-5196, 1979.

26. Shiffer KA and Goodman SR: Protein 4.1: its association with the human erythrocyte membrane. Proc Natl Acad Sci USA 81: 4404-4408, 1984.

27. Bretscher A: Purification of the intestinal microvillus cytoskeletal proteins villin, fimbrin, and ezrin. Methods Enzymol 134: 24-37, 1986.

28. Tsukita S and Hieda Y: A new $82-\mathrm{kD}$ barbed end-capping protein (radixin) localized in the cell-to-cell adherens junction: purification and characterization. J Cell Biol 108: 2369-2382, 1989.

29. Lankes WT and Furthmayr H: Moesin: a member of the protein 4.1-talin-ezrin family of proteins. Proc Natl Acad Sci USA 88: 8297-8301, 1991 .

30. Jiang WG, Hiscox S, Singhrao SK, et al: Induction of tyrosine phosphorylation and translocation of ezrin by hepatocyte growth factor/scatter factor. Biochem Biophys Res Commun 217: 1062-1069, 1995.

31. Sun CX, Robb VA and Gutmann DH: Protein 4.1 tumor suppressors: getting a FERM grip on growth regulation. J Cell Sci 115: 3991-4000, 2002

32. Takeuchi K, Kawashima A, Nagafuchi A and Tsukita S: Structural diversity of band 4.1 superfamily members. J Cell Sci 107: 1921-1928, 1994.

33. Conboy J, Kan YW, Shohet SB and Mohandas N: Molecular cloning of protein 4.1, a major structural element of the human erythrocyte membrane skeleton. Proc Natl Acad Sci USA 83: 9512-9516, 1986.

34. Smith WJ, Nassar N, Bretscher A, Cerione RA and Karplus PA: Structure of the active N-terminal domain of Ezrin. Conformational and mobility changes identify keystone interactions. J Biol Chem 278: 4949-4956, 2003.

35. Shimizu T, Seto A, Maita N, Hamada K, Tsukita S and Hakoshima T: Structural basis for neurofibromatosis type 2 . Crystal structure of the merlin FERM domain. J Biol Chem 277: 10332-10336, 2002.

36. Pearson MA, Reczek D, Bretscher A and Karplus PA: Structure of the ERM protein moesin reveals the FERM domain fold masked by an extended actin binding tail domain. Cell 101: 259-270, 2000

37. Gautreau A, Louvard D and Arpin M: ERM proteins and NF2 tumor suppressor: the Yin and Yang of cortical actin organization and cell growth signaling. Curr Opin Cell Biol 14: 104-109, 2002.

38. Bretscher A: Purification of an 80,000-dalton protein that is a component of the isolated microvillus cytoskeleton, and its localization in nonmuscle cells. J Cell Biol 97: 425-432, 1983.

39. Franck Z, Gary R and Bretscher A: Moesin, like ezrin, colocalizes with actin in the cortical cytoskeleton in cultured cells, but its expression is more variable. J Cell Sci 105: 219-231, 1993.

40. Sato N, Funayama N, Nagafuchi A, Yonemura S, Tsukita S and Tsukita S: A gene family consisting of ezrin, radixin and moesin. Its specific localization at actin filament/plasma membrane association sites. J Cell Sci 103: 131-143, 1992.

41. Louvet-Vallee S: ERM proteins: From cellular architecture to cell signaling. Biol Cell 92: 305-316, 2000.

42. Nowak D, Mazur AJ, Popow-Wozniak A, Radwanska A, Mannherz HG and Malicka-Blaszkiewicz M: Subcellular distribution and expression of cofilin and ezrin in human colon adenocarcinoma cell lines with different metastatic potential. Eur J Histochem 54: 14, 2010.

43. Sarrio D, Rodriguez-Pinilla SM, Dotor A, Calero F, Hardisson D and Palacios J: Abnormal ezrin localization is associated with clinicopathological features in invasive breast carcinomas. Breast Cancer Res Tr 98: 71-79, 2006.

44. Lankes W, Griesmacher A, Grunwald J, Schwartzalbiez R and Keller R: A heparin-binding protein involved in inhibition of smooth-muscle cell proliferation. Biochem J 251: 831-842, 1988.

45. Amieva MR and Furthmayr H: Subcellular localization of moesin in dynamic filopodia, retraction fibers, and other structures involved in substrate exploration, attachment, and cell-cell contacts. Exp Cell Res 219: 180-196, 1995.

46. Lallemand D and Arpin M: Moesin/ezrin: a specific role in cell metastasis? Pigm Cell Melanoma Res 23: 6-7, 2010.

47. He M, Cheng Y, Li W, et al: Vascular endothelial growth factor C promotes cervical cancer metastasis via up-regulation and activation of RhoA/ROCK-2/moesin cascade. BMC Cancer 10: 2010 . 
48. Amieva MR, Wilgenbus KK and Furthmayr H: Radixin is a component of hepatocyte microvilli in situ. Exp Cell Res 210: 140-144, 1994.

49. Hamada K, Shimizu T, Matsui T, Tsukita S and Hakoshima T: Structural basis of the membrane-targeting and unmasking mechanisms of the radixin FERM domain. Embo J 19 4449-4462, 2000

50. Loebrich S, Bahring R, Katsuno T, Tsukita S and Kneussel M: Activated radixin is essential for GABAA receptor alpha5 subunit anchoring at the actin cytoskeleton. EMBO J 25 . 987-999, 2006

51. Elliott BE, Meens JA, SenGupta SK, Louvard D and Arpin M: The membrane cytoskeletal crosslinker ezrin is required for metastasis of breast carcinoma cells. Breast Cancer Research 7: 365-373, 2005

52. Khanna C, Wan XL, Bose S, et al: The membrane-cytoskeleton linker ezrin is necessary for osteosarcoma metastasis. Nat Med 10: 182-186, 2004

53. Yu YL, Khan J, Khanna C, Helman L, Meltzer PS and Merlino G: Expression profiling identifies the cytoskeletal organizer ezrin and the developmental homeoprotein Six-1 as key metastatic regulators. Nat Med 10: 175-181, 2004.

54. Kang YK, Hong SW, Lee H and Kim WH: Prognostic implications of ezrin expression in human hepatocellular carcinoma. Mol Carcinog 49: 798-804, 2010.

55. Deng XY, Tannehill-Gregg SH, Nadella MVP, et al: Parathyroid hormone-related protein and ezrin are up-regulated in human lung cancer bone metastases. Clin Exp Metastas 24: 107-119, 2007.

56. Meng YX, Lu ZH, Yu SN, Zhang QA, Ma YH and Chen J: Ezrin promotes invasion and metastasis of pancreatic cancer cells. J Transl Med 8: 2010.

57. Federici C, Brambilla D, Lozupone F, et al: Pleiotropic function of ezrin in human metastatic melanomas. Int J Cancer 124: 2804-2812, 2009.

58. Zhou BB, Leng J, Hu M, et al: Ezrin is a key molecule in the metastasis of MOLT4 cells induced by CCL25/CCR9. Leuk Res 34: 769-776, 2010.

59. Morales FC, Molina JR, Hayashi Y and Georgescu MM: Overexpression of ezrin inactivates NF2 tumor suppressor in glioblastoma. Neuro Oncol 12: 528-539, 2010.

60. Cui YZ, Wu JM, Zong MJ, et al: Proteomic profiling in pancreatic cancer with and without lymph node metastasis. Int J Cancer 124: 1614-1621, 2009.

61. Estecha A, Sanchez-Martin L, Puig-Kroger A, et al: Moesin orchestrates cortical polarity of melanoma tumour cells to initiate 3D invasion. J Cell Sci 122: 3492-3501, 2009.

62. Jensen PV and Larsson LI: Actin microdomains on endothelial cells: association with CD44, ERM proteins, and signaling molecules during quiescence and wound healing. Histochem Cell Biol 121: 361-369, 2004

63. Ng T, Parsons M, Hughes WE, et al: Ezrin is a downstream effector of trafficking PKC-integrin complexes involved in the control of cell motility. EMBO J 20: 2723-2741, 2001.

64. Haas MA, Vickers JC and Dickson TC: Binding partners L1 cell adhesion molecule and the ezrin-radixin-moesin (ERM) proteins are involved in development and the regenerative response to injury of hippocampal and cortical neurons. Eur J Neurosci 20: 1436-1444, 2004.

65. Haas MA, Vickers JC and Dickson TC: Rho kinase activates ezrin-radixin-moesin (ERM) proteins and mediates thei function in cortical neuron growth, morphology and motility in vitro. J Neurosci Res 85: 34-46, 2007

66. Tsuda M, Makino Y, Iwahara T, et al: Crk associates with ERM proteins and promotes cell motility toward hyaluronic acid. J Biol Chem 279: 46843-46850, 2004

67. Crepaldi T, Gautreau A, Comoglio PM, Louvard D and Arpin M: Ezrin is an effector of hepatocyte growth factor-mediated migration and morphogenesis in epithelial cells. J Cell Biol 138 423-434, 1997.

68. Hashimoto S, Amaya F, Matsuyama H, et al: Dysregulation of lung injury and repair in moesin-deficient mice treated with intratracheal bleomycin. Am J Physiol Lung Cell Mol Physiol 295: L566-L574, 2008

69. Okayama T, Kikuchi S, Ochiai T, et al: Attenuated response to liver injury in moesin-deficient mice: impaired stellate cell migration and decreased fibrosis. Biochim Biophys Acta 1782: 542-548, 2008

70. Takakuwa Y: Regulation of red cell membrane protein interactions: implications for red cell function. Curr Opin Hematol 8: $80-84,2001$
71. Holzwarth G, Yu J and Steck TL: Heterogeneity in the conformation of different protein fractions from the human erythrocyte membrane. J Supramol Struct 4: 161-168, 1976.

72. Diakowski W, Grzybek M and Sikorski AF: Protein 4.1, a component of the erythrocyte membrane skeleton and its related homologue proteins forming the protein 4.1/FERM superfamily. Folia Histochem Cyto 44: 231-248, 2006.

73. Mattagajasingh SN, Huang SC, Hartenstein JS and Benz EJ: Characterization of the interaction between protein $4.1 \mathrm{R}$ and $\mathrm{ZO}-2$. A possible link between the tight junction and the actin cytoskeleton. J Biol Chem 275: 30573-30585, 2000.

74. Yamakawa H, Ohara R, Nakajima D, Nakayama $M$ and Ohara O: Molecular characterization of a new member of the protein 4.1 family (brain 4.1) in rat brain. Mol Brain Res 74: 247-247, 1999.

75. Tchernia G, Mohandas N and Shohet SB: Deficiency of skeletal membrane protein band 4.1 in homozygous hereditary elliptocytosis. Implications for erythrocyte membrane stability. J Clin Invest 68: 454-460, 1981.

76. Shi ZT, Afzal V, Coller B, et al: Protein 4.1R-deficient mice are viable but have erythroid membrane skeleton abnormalities. J Clin Invest 103: 331-340, 1999.

77. Salomao M, Zhang XH, Yang Y, et al: Protein 4.1R-dependent multiprotein complex: New insights into the structural organization of the red blood cell membrane. Proc Natl Acad Sci USA 105: 8026-8031, 2008

78. Nunomura W and Takakuwa Y: Regulation of protein 4.1R interactions with membrane proteins by $\mathrm{Ca} 2+$ and calmodulin. Front Biosci 11: 1522-1539, 2006.

79. Pinder JC, Gardner B and Gratzer WB: Interaction of protein 4.1 with the red cell membrane: effects of phosphorylation by protein kinase C. Biochem Biophys Res Commun 210: 478-482, 1995.

80. Horne WC, Prinz WC and Tang EK: Identification of two cAMP-dependent phosphorylation sites on erythrocyte protein 4.1. Biochim Biophys Acta 1055: 87-92, 1990.

81. Eder PS, Soong CJ and Tao M: Phosphorylation reduces the affinity of protein 4.1 for spectrin. Biochemistry 25: 1764-1770, 1986.

82. Krauss SW, Larabell CA, Lockett S, et al: Structural protein 4.1 in the nucleus of human cells: dynamic rearrangements during cell division. J Cell Biol 137: 275-289, 1997.

83. Mattagajasingh SN, Huang SC, Hartenstein JS, Snyder M, Marchesi VT and Benz EJ: A nonerythroid isoform of protein $4.1 \mathrm{R}$ interacts with the nuclear mitotic apparatus (NuMA) protein. J Cell Biol 145: 29-43, 1999.

84. Perez-Ferreiro CM, Luque CM and Correas I: 4.1R proteins associate with interphase microtubules in human T cells: a 4.1R constitutive region is involved in tubulin binding. J Biol Chem 276: 44785-44791, 2001

85. Krauss SW, Heald R, Lee G, et al: Two distinct domains of protein 4.1 critical for assembly of functional nuclei in vitro. J Biol Chem 277: 44339-44346, 2002.

86. Yang SM, Guo XH, Debnath G, Mohandas N and An XL: Protein 4.1R links E-cadherin/beta-catenin complex to the cytoskeleton through its direct interaction with beta-catenin and modulates adherens junction integrity. Biochim Biophys Acta 1788: 1458-1465, 2009.

87. Chen L, Hughes RA, Baines AJ, Conboy J, Mohandas N and An X: Protein 4.1R regulates cell adhesion, spreading, migration and motility of mouse keratinocytes by modulating surface expression of beta1 integrin. J Cell Sci 124: 2478-2487, 2011

88. Ruiz-Sáenz A, Kremer L, Alonso MA, Millan J and Correas I: Protein 4.1R regulates cell migration and IQGAP1 recruitment to the leading edge. J Cell Sci 124: 2529-2538, 2011.

89. Hashimoto Y, Shindo-Okada N, Tani M, Takeuchi K, Toma H and Yokota J: Identification of genes differentially expressed in association with metastatic potential of K-1735 murine melanoma by messenger RNA differential display. Cancer Res 56: 5266-5271, 1996.

90. Shimizu K, Nagamachi Y, Tani M, et al: Molecular cloning of a novel NF2/ERM/4.1 superfamily gene, ehm2, that is expressed in high-metastatic K1735 murine melanoma cells. Genomics 65 : 113-120, 2000

91. Chauhan S, Pandey R, Way JF, et al: Androgen regulation of the human FERM domain encoding gene EHM2 in a cell model of steroid-induced differentiation. Biochem Biophys Res Commun 310: 421-432, 2003

92. Cress AE and Nagle RB (eds): Cell Adhesion and Cytoskeletal Molecules in Metastasis. Series: Cancer Metastasis - Biology and Treatment. Vol. 9. Springer, Dordrecht, 2006. 
93. Hoover KB and Bryant PJ: Drosophila Yurt is a new protein-4.1-like protein required for epithelial morphogenesis. Dev Genes Evol 212: 230-238, 2002.

94. Wang J, Cai Y, Penland R, Chauhan S, Miesfeld RL and Ittmann M: Increased expression of the metastasis-associated gene Ehm2 in prostate cancer. Prostate 66: 1641-1652, 2006.

95. Schulz WA, Ingenwerth M, Djuidje CE, Hader C, Rahnenfuhrer $\mathrm{J}$ and Engers R: Changes in cortical cytoskeletal and extracellular matrix gene expression in prostate cancer are related to oncogenic ERG deregulation. BMC Cancer 10: 505, 2010.

96. Dhanasekaran SM, Barrette TR, Ghosh D, et al: Delineation of prognostic biomarkers in prostate cancer. Nature 412: 822-826, 2001.

97.Luo JH, Yu YP, Cieply K, et al: Gene expression analysis of prostate cancers. Mol Carcinog 33: 25-35, 2002.

98. Luo J, Duggan DJ, Chen Y, et al: Human prostate cancer and benign prostatic hyperplasia: molecular dissection by gene expression profiling. Cancer Res 61: 4683-4688, 2001.

99. Yu H, Ye L, Mansel RE, Zhang Y and Jiang WG: Clinical implications of the influence of Ehm 2 on the aggressiveness of breas cancer cells through regulation of matrix metalloproteinase- 9 expression. Mol Cancer Res 8: 1501-1512, 2010.

100. Bosanquet DC, Ye L, Harding KG and Jiang WG: Expressed in high metastatic cells (Ehm2) is a positive regulator of keratinocyte adhesion and motility: The implication for wound healing. J Dermatol Sci 71: 115-121, 2013.

101. Reid BJ, Li X, Galipeau PC and Vaughan TL: Barrett's oesophagus and oesophageal adenocarcinoma: time for a new synthesis. Nat Rev Cancer 10: 87-101, 2010.

102. De Minicis S, Marzioni M, Saccomanno S, et al: Cellular and molecular mechanisms of hepatic fibrogenesis leading to liver cancer. Transl Gastrointest Cancer 1: 88-94, 2011.

103. Mountford RA, Brown P, Salmon PR, Alvarenga C, Neumann CS and Read AE: Gastric cancer detection in gastric ulcer disease. Gut 21: 9-17, 1980.

104.Jess T, Rungoe C and Peyrin-Biroulet L: Risk of colorectal cancer in patients with ulcerative colitis: a meta-analysis of population-based cohort studies. Clin Gastroenterol Hepatol 10: 639-645, 2012.

105. Malka D, Hammel P, Maire F, et al: Risk of pancreatic adenocarcinoma in chronic pancreatitis. Gut 51: 849-852, 2002.
106. Kerr-Valentic MA, Samimi K, Rohlen BH, Agarwal JP and Rockwell WB: Marjolin's ulcer: modern analysis of an ancient problem. Plast Reconstr Surg 123: 184-191, 2009.

107. Pasternack GR, Anderson RA, Leto TL and Marchesi VT: Interactions between protein 4.1 and band 3 . An alternative binding site for an element of the membrane skeleton. J Biol Chem 260: 3676-3683, 1985 .

108. Hemming NJ, Anstee DJ, Mawby WJ, Reid ME and Tanner MJ: Localization of the protein 4.1-binding site on human erythrocyte glycophorins C and D. Biochem J 299: 191-196, 1994.

109. Marfatia SM, Leu RA, Branton D and Chishti AH: Identification of the protein 4.1 binding interface on glycophorin $\mathrm{C}$ and $\mathrm{p} 55$, a homologue of the Drosophila discs-large tumor suppressor protein. J Biol Chem 270: 715-719, 1995.

110. Reczek D, Berryman M and Bretscher A: Identification of EBP50: A PDZ-containing phosphoprotein that associates with members of the ezrin-radixin-moesin family. J Cell Biol 139: 169-179, 1997.

111. Nunomura W, Takakuwa Y, Tokimitsu R, Krauss SW, Kawashima $M$ and Mohandas N: Regulation of CD44protein 4.1 interaction by $\mathrm{Ca}^{+}$and calmodulin. Implications for modulation of CD44-ankyrin interaction. J Biol Chem 272: 30322-30328, 1997.

112. Heiska L, Alfthan K, Gronholm M, Vilja P, Vaheri A and Carpen O: Association of ezrin with intercellular adhesion molecule-1 and -2 (ICAM-1 and ICAM-2). Regulation by phosphatidylinositol 4, 5-bisphosphate. J Biol Chem 273: 21893-21900, 1998.

113. Darmellah A, Rucker-Martin C and Feuvray D: ERM proteins mediate the effects of $\mathrm{Na}^{+} / \mathrm{H}^{+}$exchanger (NHE1) activation in cardiac myocytes. Cardiovasc Res 81: 294-300, 2009.

114. Niggli V, Andreoli C, Roy C and Mangeat P: Identification of a phosphatidylinositol-4,5-bisphosphate-binding domain in the N-terminal region of ezrin. FEBS Lett 376: 172-176, 1995.

115. Tanaka T, Kadowaki K, Lazarides E and Sobue K: Ca2(+)dependent regulation of the spectrin/actin interaction by calmodulin and protein 4.1. J Biol Chem 266: 1134-1140, 1991.

116. Weinman EJ, Steplock D, Wade JB and Shenolikar S: Ezrin binding domain-deficient NHERF attenuates cAMP-mediated inhibition of $\mathrm{Na}(+) / \mathrm{H}(+)$ exchange in OK cells. Am J Physiol Renal Physiol 281: F374-F380, 2001. 